Pacific Journal of Mathematics

NORM DECREASING HOMOMORPHISMS BETWEEN GROUP

J. E. MeRLin AND Wilfred DenNis Pe ire 


\title{
NORM DECREASING HOMOMORPHISMS BETWEEN GROUP ALGEBRAS
}

\author{
J. E. Kerlin AND W. D. Pepe
}

The norm decreasing homomorphisms $\varphi$ of $L^{1}(F)$ into $M(G)$ for locally compact groups $F$ and $G$ have been characterized by $F$. $P$. Greenleaf using an integral representation. In this note the authors improve and unify some of the results and proofs of structure theorems in the previous literature. Necessary and sufficient conditions that $\varphi$ have a canonical factorization of a general type are expressed in terms of the extensibility of a $\varphi$-associated character on a $\varphi$-related closed normal subgroup. In particular, an explicit factorization of $\varphi$ can be obtained when either $F$ or $G$ is Abelian. Also investigated is the structure of norm decreasing homomorphisms $\varphi$ with range in $L^{1}(G)$.

With $F$ and $G$ denoting (throughout this note) locally compact Hausdorff groups, the norm decreasing homomorphisms of the group algebra $L^{1}(F)$ into the measure algebra $M(G)$ have been characterized by Glicksberg [2] and Cohen [1] for Abelian groups and in the general setting by Greenleaf [3]. The characterization obtained for nonAbelian groups is less tractable than that obtained in the Abelian case. (Compare Theorem 2.1 of [2] with Theorem 4.2.2 of [3].) In this note the authors give necessary and sufficient conditions for a nonzero norm decreasing homomorphism $\varphi: L^{1}(F) \rightarrow M(G)$ to have certain factorizations analogous to those obtained by Glicksberg [2, Theorems 2.1, 2.9] and Greenleaf [3, Theorem 5.1.5]. Furthermore, those $\varphi$ with range in $L^{1}(G)$ are investigated, and simpler proofs of Greenleaf's characterization of the epimorphisms and monomorphisms between $L^{1}$-group algebras (cf., [3, Theorem 5.2.1, Cor. 5.1.6], [4, Theorem 2.1]) are provided.

In the interest of brevity we adopt the notations and definitions of [3]. In addition, if $X$ and $Y$ are locally compact Hausdorff spaces and $\theta: X \rightarrow Y$ is continuous, then $\theta_{*}: M(X) \rightarrow M(Y)$ denotes the canonical norm decreasing linear map defined by $\left\langle\theta_{*}(\mu), f\right\rangle=$ $\langle\mu, f \circ \theta\rangle$ for all $f$ in $C_{0}(Y)$, or equivalently, by $\theta_{*}(\mu)(B)=\mu\left(\theta^{-1}(B)\right)$ for all Borel subsets, $B$, of $Y$; if $\theta: F \rightarrow G$ is a continuous homomorphism then $\theta_{*}$ is also multiplicative. If $K$ is a compact subgroup or closed normal subgroup of $G$, then $m_{G / K}$ denotes the positive left invariant measure on $G / K$ so that $m_{G}, m_{K}$, and $m_{G / K}$ are canonically related [6, Chaps. 3, 8]. $L^{1}(G / K)$ denotes the usual Lebesgue space with respect to $m_{G / K}$. If $K$ is compact, then $\pi_{K}^{*}: M(G / K) \rightarrow M(G)$ is the norm decreasing linear map defined by 
$\left\langle\pi_{K}^{*}(\mu), f\right\rangle=\left\langle\mu,\left(\pi_{K}\right)_{*}(f)\right\rangle$ for all $f$ in $\mathscr{K}(G)$, the space of continuous, $C$-valued, compactly supported, functions on $G$, where under the identification $L^{1}(G) \subset M(G), \quad\left(\pi_{K}\right)_{*} f(g K)=\int_{K} f(g k) d m_{K}(k)$ for $g \in G$. Let $\hat{G}$ denote the group of continuous homomorphisms of $G$ into the circle group $S^{1}$. If $\alpha$ is in $\hat{G}$, then $A_{\alpha}$ denotes the isometric automorphism of $M(G)$ defined by $\left\langle A_{\alpha}(\mu), f\right\rangle=\langle\alpha \mu, f\rangle=\langle\mu, \alpha f\rangle$ for all $f$ in $C_{0}(G)$.

Let $\bar{\varphi}:(M(F),(s o)) \rightarrow(M(G),(\sigma))$ denote the unique norm decreasing extension of $\varphi$; let $H_{0}=\bigcup$ \{support $\bar{\varphi}\left(\delta_{x}\right): x \in F$ \}, a subgroup of $G$; then $\bar{\varphi}\left(\delta_{e}\right)=\rho m_{K}$ where $K$ is a compact subgroup of $G$ normal in $H_{0}$ and $\rho$ is in $\hat{K}[3, \S 4.2]$. Let $\zeta: F \rightarrow H_{0} / K$ be the mapping defined by $\zeta(x)=\pi_{K}\left(\operatorname{supp} \bar{\varphi}\left(\delta_{x}\right)\right)$. The continuity properties of $\bar{\varphi}$ show that $\zeta$ is an epimorphism of $F$ onto $H_{0} / K$ and is continuous as a map of $F$ into $G / K$. According to [3, Lemma 5.1.2] there is a unique topology $\tau$ on $H_{0}$ making $H=\left(H_{0}, \tau\right)$ into a locally compact group, $K$ a compact subgroup of $H$, the monomorphic inclusion $j: H \rightarrow G$ continuous, and the algebraic epimorphism $\zeta$ a continuous open epimorphism $\theta: F \rightarrow H / K$. (Algebraically, $\zeta$ and $\theta$ are the same map, but their topological properties differ.) Finally, recall ([3, $\S 4.2])$ that $\bar{\varphi}\left(\delta_{x}\right)=\lambda_{x, g} \delta_{g} * \rho m_{K}$, where $\pi_{K}(g)=\zeta(x)$ for $g$ in $H_{0}$ and $\left|\lambda_{x, g}\right|=1$.

LeMmA 1. Let $F_{\varphi}=\left\{x \in F: \bar{\phi}\left(\delta_{x}\right)=\lambda_{x} \rho m_{K}\right.$ for some $\left.\left|\lambda_{x}\right|=1\right\}$, and let $\gamma_{\varphi}: F_{\varphi} \rightarrow S^{1}$ be defined by $\gamma_{\varphi}(x)=\lambda_{x}$. Then $F_{\varphi}$ is a closed normal subgroup with $\operatorname{Ker} \zeta=F_{\varphi}$ and $\gamma_{\varphi}$ is in $\hat{F}_{\varphi}$.

Proof. $x \in \mathrm{F}_{\varphi}$ iff $\bar{\varphi}\left(\delta_{x}\right)=\lambda_{x} \delta_{e} * \rho m_{K}=\lambda_{x, e} \delta_{e} * \rho m_{K}$ iff $\lambda_{x}=\lambda_{x, e}$ and $\zeta(x)=\pi_{K}(e)=e / K$. Thus, $F_{\varphi}=\operatorname{Ker} \zeta$ and, therefore, it is a closed normal subgroup. As $\varphi$ is a homomorphism and is ((so), ( $\sigma)$ )continuous on norm bounded sets, it is easy to see that $\gamma_{\varphi}$ is in $\hat{F}_{\varphi}$.

We now state the main results reserving their proofs till later.

THEOREM 1. (i) $\gamma_{\varphi}$ has an extension to a character $\gamma$ in $\hat{F}$ iff $\varphi=j_{*} A_{\beta} \pi_{K}^{*} \theta_{*} A_{r}$ where $K$ is a compact normal subgroup of a locally compact group $H, \theta: F \rightarrow H / K$ is a continuous open epimorphism, $j: H \rightarrow G$ is a continuous monomorphism, and $\gamma \in \hat{F}, \beta \in \hat{H}$.

(ii) $\rho$ has an extension to a character $\alpha$ in $\hat{G}$ iff $\varphi=A_{\alpha} \pi_{K}^{*} \zeta_{*} A_{r}$ where $K$ is a compact subgroup normal in a subgroup $H_{0}$ of $G$, $\zeta: F \rightarrow H_{0} / K \subset G / K$ is a continuous epimorphism, and $\gamma \in \hat{F}, \alpha \in \hat{G}$.

TheOREM 2. Let $H$ and $G$ be locally compact groups, let $K \subset H$ and $J \subset G$ be compact subgroups, let $L$ be a subgroup of $G$ with $J$ 
normal in $L$, let $\psi: H \rightarrow L / J \subset G / J$ be a continuous epimorphism with the relative topology on $L / J$, and let $\beta \in \hat{H}$ with $\operatorname{Ker} \beta \supset \operatorname{Ker} \psi$. Then the following are equivalent:

(i) $L$ is an open/closed subgroup of $G$ and $\psi$ is an open map;

(ii) $\psi_{*}\left(L^{1}(H)\right) \subset L^{1}(G / J)$;

(iii) $\psi_{*} A_{\beta} \pi_{K}^{*}\left(L^{1}(H / K)\right) \subset L^{1}(G / J)$;

(iv) $\psi^{-1}$ satisfies property $N_{\text {loc }}$, i.e., the $\psi$-inverse image of an $m_{G / J}$-locally null set in $G / J$ is $m_{H}$-locally null in $H$; and,

(v) if $H$ and $G$ are Abelian, then the dual homomorphism $\hat{\psi}:(G / J)^{\wedge} \rightarrow \hat{H}$ is a proper map, i.e., the $\hat{\psi}$-inverse image of a compact subset is compact.

CoRollary 1. (i ) $\gamma_{\varphi} \equiv 1$ iff $\varphi=j_{*} A_{\beta} \pi_{K}^{*} \theta_{*}$.

(ii) $\bar{\varphi}\left(\delta_{e}\right) \geqq 0$ iff $\varphi=\pi_{K}^{*} \zeta_{*} A_{\gamma}$.

(iii) $\varphi$ is order preserving iff $\varphi=\pi_{K}^{*} \zeta_{*}$.

(iv) $\langle\varphi(f), \bar{\alpha}\rangle \neq 0$ for some $f$ in $L^{1}(F)$ and some $\alpha$ in $\hat{G}$ iff $\varphi=A_{\alpha} \pi_{K}^{*} \zeta_{*} A_{\gamma}$.

Proof. (i), (ii) and (iii) are all obvious from Theorem 1. If $\langle\varphi(f), \alpha\rangle \neq 0$ for some $f$ and $\alpha$, then the $\operatorname{map} f \rightarrow\langle\varphi(f), \bar{\alpha}\rangle$ is a nonzero multiplicative linear functional on $L^{1}(F)$ and hence ([5, (23.7)]) there is a $\gamma$ in $\hat{F}$ such that $\langle\varphi(f), \bar{\alpha}\rangle=\langle f, \gamma\rangle$ for all $f$ in $L^{1}(F)$. Let $f \geqq 0$ be in $L^{1}(F)$ and $\|f\|_{1}=1$. Then $\left\langle A_{\alpha}^{-1} \varphi A_{\gamma}^{-1}(f), 1\right\rangle=$ $\left.\left\langle\varphi A_{\tau}^{-1}(f)\right\rangle, \bar{\alpha}\right\rangle=\langle\bar{\gamma} f, \gamma\rangle=\|f\|_{1}=1$. As $\left\|A_{\alpha}^{-1} \varphi A_{\gamma}^{-1}(f)\right\|_{1} \leqq 1$, it must be that $A_{\alpha}^{-1} \varphi A_{\gamma}^{-1}(f) \geqq 0$ and hence $A_{\alpha}^{-1} \varphi A_{\gamma}^{-1}$ is order preserving. Thus $\varphi=A_{\alpha} \pi_{K}^{*} \zeta_{*} A_{r}$. Conversely, if $\varphi=A_{\alpha} \pi_{K}^{*} \zeta_{*} A_{\gamma}$, then for all $g \geqq 0$ in $L^{1}(F)$ and with $f=\bar{\gamma} g$, we have $\langle\varphi(f), \bar{\alpha}\rangle=\left\langle\pi_{K}^{*} \zeta_{*}(g), 1\right\rangle$ and this is clearly nonzero for some $g \geqq 0$.

CoROllaRY 2. (i) If $F$ is Abelian, then every nonzero $\varphi: L^{1}(F) \rightarrow M(G)$ is of the form $\varphi=j_{*} A_{\beta} \pi_{K}^{*} \theta_{*} A_{r}$.

(ii) If $G$ is Abelian, then every nonzero $\varphi: L^{1}(F) \rightarrow M(G)$ is of the form $\varphi=A_{\alpha} \pi_{K}^{*} \zeta_{*} A_{r}$.

Proof. This follows immediately from Theorem 1 since it is well known that characters on closed subgroups of Abelian groups extend to the whole group.

CoRollary 3. (i) $\varphi=j_{*} A_{\beta} \pi_{K}^{*} \theta_{*} A_{r}$ maps $L^{1}(F)$ into $L^{1}(G)$ iff $j(H)$ is open/closed in $G$ and $j: H \rightarrow G$ is an open map.

(ii) $\varnothing=A_{\alpha} \pi_{K}^{*} \zeta_{*} A_{r}$ maps $L^{1}(F)$ into $L^{1}(G)$ iff $H_{0}$ is an open/closed subgroup of $G$ and $\zeta: F \rightarrow G / K$ is an open mapping.

Proof. To prove (i), note that as $\theta_{*} A_{r}\left(L^{1}(F)\right)=L^{1}(H / K)$, we 
have $j_{*} A_{\beta} \pi_{K}^{*}\left(L^{1}(H / K)\right) \subset L^{1}(G)$ if $\varphi\left(L^{1}(F)\right) \subset L^{1}(G)$ and so Theorem 2 applies with $\psi=j, L=H_{0}$, and $J=(e)$; the converse of (i) is well known. If $\varphi=A_{\alpha} \pi_{K}^{*} \zeta_{*} A_{\gamma}$ maps $L^{1}(F)$ into $L^{1}(G)$, then $\pi_{K}^{*} \zeta_{*}\left(L^{1}(F)\right) \subset$ $L^{1}(G)$. Since $\left(\pi_{K}\right)_{*} \circ \pi_{K}^{*}$ is the identity map, we have $\zeta_{*}\left(L^{1}(F)\right) \subset$ $\left(\pi_{K}\right)_{*}\left(L^{1}(G)\right) \subset L^{1}(G / K)$. Now, Theorem 2 applies with $H=F, J=K$, $L=H_{0}$, and $\psi=\zeta$; again, the converse of (ii) is well known.

An immediate corollary to Corollary 3 (i) is

CoRollary 4 (Greenleaf [4, Theorem 2.1]). $\varphi$ is a monomorphism of $L^{1}(F)$ into $L^{1}(G)$ iff $\varphi=j_{*} A_{\beta} \pi_{K}^{*} \theta_{*}$ where $\theta: F \cong H / K$ and $j$ is a topological isomorphism of $H$ onto an open/closed subgroup of $G$.

CoRollary 5 (Greenleaf [3, Theorem 5.2.1]). $\varphi$ is an epimorphism of $L^{1}(F)$ onto $L^{1}(G)$ iff $\varphi=\Lambda T_{F_{0}} A_{\gamma}$ where $F_{0}$ is a closed normal subgroup of $F, T_{F_{0}}=\left(\pi_{F_{0}}\right)_{L^{\top}\left(F^{\top}\right)}, \quad \gamma \in \hat{F}$, and $\Lambda: L^{1}\left(F / F_{0}\right) \cong L^{1}(G)$ is an isometric isomorphism.

Proof. If $\varphi$ is an epimorphism, then $\langle\varphi(f), 1\rangle \neq 0$ for some $f$ in $L^{1}(F)$ and so $\varphi=\pi_{K}^{*} \zeta_{*} A_{\gamma}$ by Corollary 1 (iv). By Corollary 3 (ii), $\zeta$ is an open map and $H_{0}$ is an open/closed subgroup of $G$. Since elements in $\pi_{K}^{*}\left(L^{1}(G / K)\right)$ are constant on the cosets of $K$ in $G$ and $\varphi$ is an epimorphism, we must have $K=\{e\}$ and $\varphi=\zeta_{*} A_{r}$. Since $\zeta$ maps onto the open/closed subgroup $H_{0}$, it follows immediately from the definition of $\zeta_{*}$ that $\zeta_{*}\left(L^{1}(F)\right)$ is supported on $H_{0}$ in $G$. However, $\zeta_{*}\left(L^{1}(F)\right)=\zeta_{*}\left(A_{\gamma}\left(L^{1}(F)\right)\right)=\varphi\left(L^{1}(F)\right)=L^{1}(G)$ and hence $H_{0}=G$ and $\zeta: F \rightarrow G$ is a continuous open epimorphism. Let $\zeta=\lambda \circ \pi_{F_{0}}$ where $F_{0}=\operatorname{Ker} \zeta$ and $\lambda: F / F_{0} \cong G$. Then $\varphi=\zeta_{*} A_{r}=\lambda_{*} T_{F_{0}} A_{r}$ where $\lambda_{*}: L^{1}\left(F / F_{0}\right) \cong L^{1}(G)$.

The next corollary is an interesting parallel of Corollary 1.2 and Theorem 2.3 of [4].

Corollary 6. (i) (Greenleaf) $L^{1}(F)$ has a nonzero norm decreasing homomorphic image in $M(G)$ iff $F / F_{0} \cong H / K$ where $F_{0}$ is closed normal subgroup of $F, K$ is a compact normal subgroup of a locally compact group $H$, and $H$ is continuously isomorphic to a (not necessarily closed) subgroup of $G$.

(ii) $L^{1}(F)$ has a nonzero norm decreasing homomorphic image in $L^{1}(G)$ of either of the types described in Theorem 1 iff $F / F_{0} \cong$ $H / K$ where $F_{0}$ is a closed normal subgroup of $F$ and $K$ is a compact normal subgroup of a locally compact group $H$ which is topologically isomorphic to an open/closed subgroup of $G$.

Proof. Part (i) is immediate from the construction of $H=\left(H_{0}, \tau\right)$ 
and $\theta: F \rightarrow H / K$. Part (ii) follows from Corollary 3.

It is clear that if $\varphi=A_{\alpha} \pi_{K}^{*} \zeta_{*} A_{r}$, then $\varphi=j_{*} A_{\beta} \pi_{K}^{*} \theta_{*} A_{r}$ where $\beta=\alpha \circ j$, and so $j_{*} A_{\beta} \pi_{K}^{*} \theta_{*} A_{r}$ is the more general factorization of the two types. There naturally arises the question of existence of a $\varphi$ not of this general form. In light of Theorem 1 and its corollaries, an example has been difficult to find and has eluded the authors. The supporting evidence is favorable in view of the well known fact that in general characters on closed subgroups need not extend to the whole group.

Proof of Theorem 1. Part (i). If $\phi$ has the indicated factorization, then $\bar{\varphi}=j_{*} A_{\beta} \pi_{K}^{*} \theta_{*} A_{\gamma}$ and

$$
\bar{\varphi}\left(\delta_{x}\right)=\beta(g) \gamma(x) \delta_{j(g)} *\left(\beta \circ j^{-1}\right) m_{j(K)},
$$

where $\pi_{K}(g)=\theta(x)$ for $x$ in $F$ and $g$ in $H$. It follows that $i=$ $\left(\beta \circ j^{-1}\right) m_{j(K)}$ is the unit of $\Gamma=\bar{\varphi}\left(\left\{\delta_{x}: x \in F\right\}\right)$, and $\bar{\varphi}\left(\delta_{x}\right)=\lambda_{x} i$ for $\left|\lambda_{x}\right|=1$ iff $\bar{\varphi}\left(\delta_{x}\right)=\gamma(x) i$. Thus, $\gamma(x)=\gamma_{\varphi}(x)$ on $F_{\varphi}$. Conversely, suppose $\gamma_{\varphi}$ has an extension to a character $\gamma$ in $\hat{F}$. Let $\psi=\varphi \circ A_{\gamma}^{-1}$, a norm decreasing homomorphism of $L^{1}(F)$ into $M(G)$ with $\bar{\psi}=\bar{\varphi} A_{\tau}^{-1}$. Since $\bar{\psi}\left(\delta_{x}\right)=\overline{\gamma(x)} \bar{\varphi}\left(\delta_{x}\right)$ for all $x$ in $F$, we have that $\bigcup\left\{\operatorname{supp} \bar{\psi}\left(\delta_{x}\right)\right.$ : $\mathrm{x} \in F\}=H_{0}, \bar{\psi}\left(\delta_{e}\right)=\bar{\varphi}\left(\delta_{e}\right)=\rho m_{K}=i$, and $\bar{\varphi}$ and $\bar{\psi}$ determine the same $\zeta: F \rightarrow H_{0} / K \subset G / K$ and $\theta: F \rightarrow H / K$. Furthermore, if $\bar{\psi}\left(\delta_{x}\right)=\lambda i$ for some $|\lambda|=1$, then $\gamma(x) \lambda i=\gamma(x) \bar{\psi}\left(\delta_{x}\right)=\bar{\varphi}\left(\delta_{x}\right)$. Therefore, $x \in F_{\varphi}$ and $\gamma_{\varphi}(x)=\gamma(x) \lambda$. Since $\gamma_{\varphi}$ is the restriction of $\gamma$ to $F_{\varphi}, \lambda=1$. Thus, $\bar{\psi}\left(\left\{\delta_{x}: x \in F\right\}\right) \cap S^{1} i=\{i\}$ and by Theorem 5.1.5 of [3], $\psi=j_{*} A_{\beta} \pi_{K}^{*} \theta_{*}$; therefore, $\varphi=\psi A_{r}=j_{*} A_{\beta} \pi_{K}^{*} \theta_{*} A_{r}$.

Part (ii). If $\rho$ has an extension to a character $\alpha$ in $\hat{G}$, consider $\psi=A_{\alpha}^{-1} \varphi$, a norm decreasing homomorphism of $L^{1}(F)$ into $M(G)$ with $\bar{\psi}=A_{\alpha}^{-1} \bar{\varphi}$. Since $\alpha$ extends $\rho$ we have for each $x$ in $F, \bar{\psi}\left(\delta_{x}\right)=$ $A_{a}^{-1}\left(\lambda_{x, g} \delta_{g} * \rho m_{K}\right)=\left(\lambda_{x, g} \overline{\alpha(g)}\right) \delta_{g} * m_{K}$. Thus, $\bar{\varphi}$ and $\bar{\psi}$ determine the same compact $K$, subgroup $H_{0}$ in $G$, and maps $\zeta$ and $\theta$. Let $\eta_{x, g}=$ $\lambda_{x, g} \overline{\alpha(g)}$ so that $\bar{\psi}\left(\delta_{x}\right)=\eta_{x, g} \delta_{g} * m_{K}$ where $\pi_{K}(g)=\zeta(x)$. Since $\delta_{g} * m_{K}$ does not depend on the representative $g$ in the coset $g K, \gamma(x)=\eta_{x, g}$ is a well defined function on $F$ to $S^{1}$. It follows easily from the continuity properties of $\bar{\psi}$ that $\gamma \in \hat{F}$; moreover, $\gamma$ extends $\gamma \psi$. Now, the map $\pi_{K}^{*} \zeta_{*} A_{\gamma}$ agrees with $\bar{\psi}$ on $\left\{\lambda \delta_{x}: x \in F,|\lambda|=1\right\}=S^{1} \xi_{F}$. As $\pi_{K}^{*} \zeta_{*} A_{\gamma}$ and $\bar{\psi}$ are ((so), $\left.(\sigma)\right)$-continuous on norm bounded sets and as co $\left[S^{1} \xi_{F}:\right.$ (so)] is the unit ball in $M(F)$ ([3, Lemma 1.1.3]), we have $\pi_{K}^{*} \zeta_{*} A_{\gamma}=\bar{\psi}$ and $\varphi=A_{\alpha} \pi_{K}^{*} \zeta_{*} A_{\gamma}$. The converse of (ii) is easily seen from the relation $A_{\alpha} \pi_{K}^{*} \zeta_{*} A_{\gamma}\left(\delta_{e}\right)=A_{\alpha}\left(m_{K}\right)=\left(\left.\alpha\right|_{K}\right) m_{K}$.

Proof of Theorem 2. As the adjoint map of the homomorphism $\psi_{*}$ is $\hat{\psi}:(G / J)^{\wedge} \rightarrow \hat{H}$ when $H$ and $G$ are Abelian, the equivalence of 
(ii) and (v) is due to P. Cohen [1, Theorem 1]. As it is well known that (i) implies (ii), and clearly (ii) implies (iii), it is necessary only to prove (iii) implies (iv) and (iv) implies (i).

Assume (iii). We first show $\psi_{*} \pi_{K}^{*}\left(L^{1}(H / K)\right) \subset L^{1}(G / J)$. Let $f$ be in $\mathscr{C}(H / K)$, and let $C=\pi_{K}^{-1}(\operatorname{supp} f)$, a compact subset of $H$. Let $\beta^{\sim}$ denote the bounded Borel function on $G / J$ defined by $\beta^{\sim}=0$ outside of $\psi(C)$ and $\beta^{\sim}(x)=\overline{\beta(y)}$ for $x \in \psi(C)$ and $\psi(y)=x, y \in H$. Since $\operatorname{Ker} \psi \subset \operatorname{Ker} \beta, \beta^{\sim}$ is well defined on the compact subset $\psi(C)$ and it is continuous on $\psi(C)$. Let $A_{\beta}: L^{1}(G / J) \rightarrow L^{1}(G / J)$ denote the bounded linear map of pointwise multiplication by $\beta^{2}$. Now, since $\beta^{\sim} \circ \psi=\bar{\beta}$ on $C, \pi_{K}^{*}(f)=f \circ \pi_{K}$ is supported on $C$, and $\psi_{*} A_{\beta} \pi_{K}(f) \epsilon$ $L^{1}(G / J)$, we have $\left\langle A_{\beta} \sim \psi_{*} A_{\beta} \pi_{K}^{*}(f), g\right\rangle=\left\langle\pi_{K}^{*}(f), \beta\left(\beta^{\sim} \circ \psi\right)(g \circ \psi)\right\rangle=\left\langle\pi_{K}^{*}(f)\right.$, $g \circ \psi\rangle=\left\langle\psi_{*} \pi_{K}^{*}(f), g\right\rangle$ for all $g$ in $C_{0}(G / J)$. Therefore, $A_{\beta} \sim \psi_{*} A_{\beta} \pi_{K}^{*}(f)=$ $\psi_{*} \pi_{K}^{*}(f)$ and it is in $L^{1}(G / J)$. Since $f$ in $\mathscr{K}(H / K)$ is arbitrary and $\psi_{*} \pi_{K}^{*}$ is continuous, we have that $\psi_{*} \pi_{K}^{*}$ maps $L^{1}(H / K)$ into $L^{1}(G / J)$.

To prove (iv), first consider any Borel $m_{G / J}$-null set $B$ in $G / J$, and let $C$ be any compact subset of $H$ with $C \cdot K=C$. Now, as $\chi_{C}=\pi_{K}^{*}\left(\chi_{\pi_{K^{(}(C)}}\right)$, as $\psi_{*} \pi_{K}^{*}\left(\chi_{\left.\pi_{K^{(}(C)}\right)}\right)$ is in $L^{1}(G / J)$, and as $B$ is a null set, we have $m_{H}\left(C \cap \psi^{-1}(B)\right)=\chi_{C} d m_{H}\left(\psi^{-1}(B)\right)=\psi_{*} \pi_{K}^{*}\left(\chi_{\pi_{K}(C)}\right)(B)=0$. Thus, $C \cap \psi^{-1}(B)$ is an $m_{H^{-}}$-null set. Since every compact subset of $H$ is contained in a compact set $C$ where $C \cdot K=C$, it follows that $\psi^{-1}(B)$ is $m_{H}$-locally null and hence $\psi^{-1}(M)$ is $m_{H^{-}}$-locally null for any $m_{G / J^{-}}$ null set $M$ in $G / J$. Finally, let $N$ be any $m_{G / J}$-locally null set in $G / J$ and let $C$ be any compact subset of $H$. Since $C \cap \psi^{-1}(\psi(C) \cap N)=$ $C \cap \psi^{-1}(N)$, and since $\psi(C) \cap N$ is $m_{G / J}$-null, we have that $C \cap \psi^{-1}(N)$ is $m_{H}$-null. Thus, $\psi^{-1}(N)$ is $m_{H}$-locally null and (iv) holds.

Assume (iv). As every locally compact group is the union of $\sigma$-compact open subgroups, it suffices (in order to prove (i)) to show $\psi(S)$ is open in $G / J$ and $\left.\psi\right|_{S}$ is an open map for any $\sigma$-compact open subgroup $S$ in $H$. A theorem of Pontryagin (cf., [5, (5.29)]) shows that any continuous epimorphism between $\sigma$-compact locally compact groups is an open map. Therefore, it suffices to show $\psi(S)$ is open in $G / J$ and hence a ( $\sigma$-compact) locally compact group with the relative topology. It is clear that $\psi(S)$ is at least $\sigma$-compact and therefore measurable. Since the restriction of the Haar measure $m_{H}$ to the open subgroup $S$ is the Haar measure on $S$, it is clear that $\left(\left.\psi\right|_{S}\right)^{-1}$ satisfies property $N_{\text {loc }}$. Thus $\psi(S)$ is not $m_{G / J}$-locally null. Let $L_{S}=\pi_{J}^{-1}(\psi(S))$, an $F_{\sigma}$-subgroup of $G$. By $[6, \S 3.9$, p. 66 and $\S 2.2$, p. 165] $L_{S}$ is $m_{G}$-locally null iff $\psi(S)$ is $m_{G / J}$-locally null. Therefore $L_{S}$ is not $m_{G}$-locally null and thereby contains a Borel subset $A$ of positive finite measure. Then $A A^{-1}$, a subset of $L_{S}$, is a neighborhood of the identity of $G$ [5, (20.17)], and so $L_{S}$ and $\pi_{J}\left(L_{S}\right)=\psi(S)$ are open in $G$ and $G / J$, respectively. The proof is complete. 


\section{REFERENCES}

1. P. J. Cohen, On homomorphisms of group algebras, Amer. J. Math., 82 (1960), 213-226.

2. I. Glicksberg, Homomorphisms of certain algebras of measures, Pacific J. Math., 10 (1960), 167-191.

3. F. P. Greenleaf, Norm decreasing homomorphisms of group algebras, Pacific J. Math., 15 (1965) 1187-1219.

4. - Subalgebras of group algebras, Function Algebras, F. T. Birtel editor, Scott, Foresman, Chicago, 1965.

5. E. Hewitt and K. A. Ross, Abstract Harmonic Analysis I, Springer-Verlag, Berlin, 1963.

6. H. Reiter, Classical Harmonic Analysis and Locally Compact Groups, Oxford Univ. Press, London, 1968.

Received December 27, 1973.

UNIVERSITY OF KENTUCKY-LEXINGTON 



\section{PACIFIC JOURNAL OF MATHEMATICS}

\section{EDITORS}

RICHARD ARENS (Managing Editor)

University of California

Los Angeles, California 90024

\section{J. DugundJI}

Department of Mathematics University of Southern California Los Angeles, California 90007

D. Gilbarg and J. Milgram

Stanford University

Stanford, California 94305
University of Washington Seattle, Washington 98105

\section{ASSOCIATE EDITORS}
E. F. BECKENBACH
B. H. NeumanN
F. WolF
K. YoShIDA

\section{SUPPORTING INSTITUTIONS}

\author{
UNIVERSITY OF SOUTHERN CALIFORNIA \\ STANFORD UNIVERSITY \\ UNIVERSITY OF TOKYO \\ UNIVERSITY OF UTAH \\ WASHINGTON STATE UNIVERSITY \\ UNIVERSITY OF WASHINGTON \\ $\stackrel{*}{*} \stackrel{*}{*} \stackrel{*}{ }{ }^{*}$ AMERICAN MATHEMATICAL SOCIETY
}

The Supporting Institutions listed above contribute to the cost of publication of this Journal, but they are not owners or publishers and have no responsibility for its content or policies.

Mathematical papers intended for publication in the Pacific Journal of Mathematics should be in typed form or offset-reproduced, (not dittoed), double spaced with large margins. Underline Greek letters in red, German in green, and script in blue. The first paragraph or two must be capable of being used separately as a synopsis of the entire paper. Items of the bibliography should not be cited there unless absolutely necessary, in which case they must be identified by author and Journal, rather than by item number. Manuscripts, in triplicate, may be sent to any one of the editors. Please classify according to the scheme of Math. Reviews, Index to Vol. 39. All other communications should be addressed to the managing editor, or Elaine Barth, University of California, Los Angeles, California, 90024.

The Pacific Journal of Mathematics expects the author's institution to pay page charges, and reserves the right to delay publication for nonpayment of charges in case of financial emergency.

100 reprints are provided free for each article, only if page charges have been substantially paid. Additional copies may be obtained at cost in multiples of 50 .

The Pacific Journal of Mathematics is issued monthly as of January 1966. Regular subscription rate: $\$ 72.00$ a year (6 Vols., 12 issues). Special rate: $\$ 36.00$ a year to individual members of supporting institutions.

Subscriptions, orders for back numbers, and changes of address should be sent to Pacific Journal of Mathematics, 103 Highland Boulevard, Berkeley, California, 94708.

\section{PUBLISHED BY PACIFIC JOURNAL OF MATHEMATICS, A NON-PROFIT CORPORATION}

Printed at Kokusai Bunken Insatsusha (International Academic Printing Co., Ltd.), 270, 3-chome Totsuka-cho, Shinjuku-ku, Tokyo 160, Japan.

\section{Copyright (C) 1975 by Pacific Journal of Mathematics} Manufactured and first issued in Japan 


\section{Pacific Journal of Mathematics}

\section{Vol. 57, No. $2 \quad$ February, 1975}

Norman Larrabee Alling, On Cauchy's theorem for real algebraic curves with boundary .......

Daniel D. Anderson, A remark on the lattice of ideals of a Prüfer domain ..................

Dennis Neal Barr and Peter D. Miletta, A necessary and sufficient condition for uniqueness of

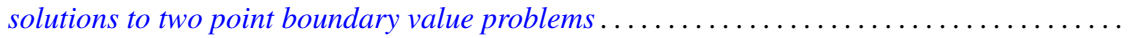

Ladislav Beran, On solvability of generalized orthomodular lattices . . . . . . . . . . ........

L. Carlitz, A three-term relation for some sums related to Dedekind sums . . . . . . . . . .....

Arthur Herbert Copeland, Jr. and Albert Oscar Shar, Images and pre-images of localization

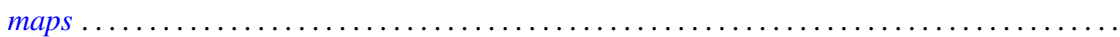

G. G. Dandapat, John L. Hunsucker and Carl Pomerance, Some new results on odd perfect

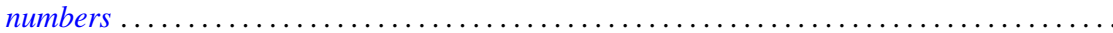

M. Edelstein and L. Keener, Characterizations of infinite-dimensional and nonreflexive

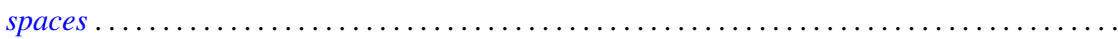

Francis James Flanigan, On Levi factors of derivation algebras and the radical embedding

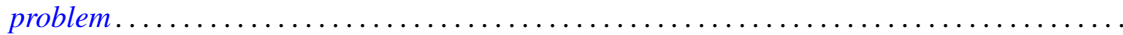

Harvey Friedman, Provable equality in primitive recursive arithmetic with and without

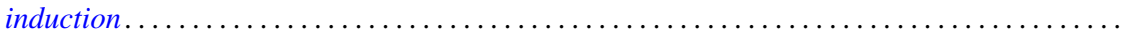

Joseph Braucher Fugate and Lee K. Mohler, The fixed point property for tree-like continua with

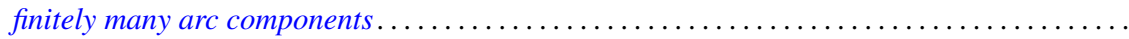

John Norman Ginsburg and Victor Harold Saks, Some applications of ultrafilters in

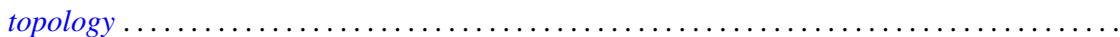

Arjun K. Gupta, Generalisation of a "square" functional equation .....................

Thomas Lee Hayden and Frank Jones Massey, Nonlinear holomorphic semigroups ..........

V. Kannan and Thekkedath Thrivikraman, Lattices of Hausdorff compactifications of a locally

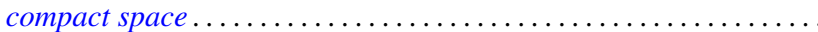

J. E. Kerlin and Wilfred Dennis Pepe, Norm decreasing homomorphisms between group

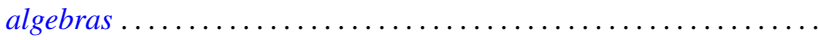

Young K. Kwon, Behavior of $\Phi$-bounded harmonic functions at the Wiener boundary ...

Richard Arthur Levaro, Projective quasi-coherent sheaves of modules .

Chung Lin, Rearranging Fourier transforms on groups...........................

David Lowell Lovelady, An asymptotic analysis of an odd order linear differential equation . . 4475

Jerry Malzan, On groups with a single involution .......................... 481

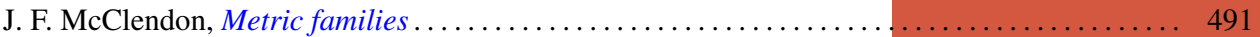

Carl Pomerance, On multiply perfect numbers with a special property .

Mohan S. Putcha and Adil Mohamed Yaqub, Polynomial constraints for finiteness of semisimple rings. .

Calvin R. Putnam, Hyponormal contractions and strong power convergence . . . . . . . . . 531

Douglas Conner Ravenel, Multiplicative operations in $\mathrm{BP} * \mathrm{BP} \ldots \ldots \ldots \ldots \ldots \ldots \ldots \ldots \ldots .539$

Judith Roitman, Attaining the spread at cardinals which are not strong limits . . . . . . . . . 545

Kazuyuki Saitô, Groups of *-automorphisms and invariant maps of von Neumann algebras . . . 553

Brian Kirkwood Schmidt, Homotopy invariance of contravariant functors acting on smooth

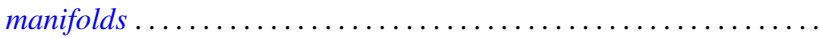

Kenneth Barry Stolarsky, The sum of the distances to $N$ points on a sphere.

Mark Lawrence Teply, Semiprime rings with the singular splitting property.

J. Pelham Thomas, Maximal connected Hausdorff spaces..............

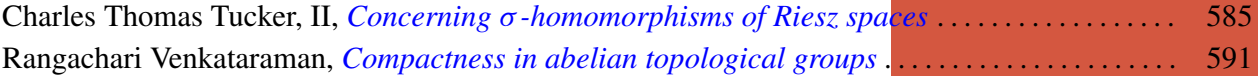

William Charles Waterhouse, Basically bounded functors and flat sheaves . . . . . . . . . . . 597

David Westreich, Bifurcation of operator equations with unbounded linearized part ......... 611

William Robin Zame, Extendibility, boundedness and sequential convergence in spaces of 